\title{
Periodic solutions for a seasonally forced SIR model with impact of media coverage
}

Jian $\mathrm{Zu}^{1}$ and Lin Wang ${ }^{2,3^{*}}$

\author{
"Correspondence: \\ wanglinmath@hotmail.com \\ ${ }^{2}$ School of Basic Science, \\ Changchun University of \\ Technology, Changchun, 130012 \\ P.R. China \\ ${ }^{3}$ College of Mathematics, Jilin \\ University, Changchun, 130012, \\ P.R. China \\ Full list of author information is \\ available at the end of the article
}

\begin{abstract}
In this paper, we study periodic solutions for a seasonally forced SIR model with impact of media coverage. Usually, media reports, information processing, and individuals' alerted responses to the information can only arise as the number of infected individuals reaches and exceeds a certain level. The piecewise smooth righthand side is introduced to describe the impact of this kind of media coverage. Using Leray-Schauder degree theory, we establish new results on the existence of at least one positive periodic solution for a seasonally forced SIR model with impact of media coverage. Some numerical simulations are presented to illustrate the effectiveness of such media coverage.
\end{abstract}

MSC: $34 \mathrm{C} 25 ; 37 \mathrm{~J} 45 ; 92 \mathrm{~B} 05$

Keywords: periodic solution; SIR model; media effects; non-smooth righthand sides

\section{Introduction}

Many infectious diseases, such as measles, chickenpox, mumps, rubella, pertussis and influenza, show seasonal patterns of incidence [1-3]. The cause of seasonal patterns may vary from the periodic contact rates [3, 4], periodic fluctuation in birth and death rates [5-7], and periodic vaccination program [8]. Thus, it is natural to model these diseases by seasonally forced epidemiological models.

The media coverage is an important factor responsible for the transmission of an infectious disease. When a type of contagious disease appears and starts to spread, people's response to the threat of disease is dependent on their perception of risk, which is affected by public and private information disseminated widely by the media. Massive news coverage and fast information flow have played an important role in affecting the outcome of infectious disease outbreak, such as the 2003 severe acute respiratory syndrome (SARS) and the 2009 H1N1 influenza epidemic [9-14].

Recently, Wang and Xiao have used piecewise continuous transmission rate to describe that the media coverage exhibits its effect once the number of infected individuals exceeds a certain critical level [15]. In this paper, we study the following periodic forced SIR model:

$$
\left\{\begin{array}{l}
\frac{d S(t)}{d t}=\mu-\beta(t) f(I) S I-\mu S, \\
\frac{d I(t)}{d t}=\beta(t) f(I) S I-(\mu+\gamma) I, \\
\frac{d R(t)}{d t}=\gamma I-\mu R,
\end{array}\right.
$$

(0) 2015 Zu and Wang; licensee Springer. This article is distributed under the terms of the Creative Commons Attribution 4.0 International License (http://creativecommons.org/licenses/by/4.0/), which permits unrestricted use, distribution, and reproduction in any medium, provided you give appropriate credit to the original author(s) and the source, provide a link to the Creative Commons license, and indicate if changes were made. 
in which

- $S, I, R$ are the fractions of the susceptible, infective and recovered population,

- $\mu$ and $\gamma$ denote the birth (death) rate and recovery rate respectively, which are positive constant,

- $\beta(t)$ is the seasonally-dependent transmission rate, which is a positive continuous $T$-periodic function,

- $f(I)$ is a decreasing piecewise smooth factor which can describe the impact of media coverage on the transmission coefficient, given by

$$
f(I)= \begin{cases}1, & I \leq I_{c} \\ 1+\sigma^{-1}\left(e^{-\alpha\left(I_{c}+\sigma\right)}-1\right)\left(I-I_{c}\right), & I_{c}<I<I_{c}+\sigma, \\ e^{-\alpha I}, & I \geq I_{c}+\sigma\end{cases}
$$

where $\alpha$ is the factor of influences, $\sigma$ is a small parameter and $I_{c}$ is a critical level.

We think that $f(I)$ in (1.2) is a good approximation to the discontinuous factor in [15] provided $\sigma$ is small enough. Denote the basic reproduction number $\mathcal{R}_{0}=\frac{\bar{\beta}}{\gamma+\mu}$ with $\bar{\beta}=\frac{1}{T} \int_{0}^{T} \beta(t) d t$. When $f(I) \equiv 1$ in (1.1), Katriel [16] got the existence of periodic positive solutions for the periodically forced SIR model by Leray-Schauder degree theory provided $\mathcal{R}_{0}>1$. By Gaines-Mawhin's continuation theorem, Jódar et al. [17] obtained that a $T$ periodic solution exists for a more general system whenever the condition $\min _{t \in \mathbb{R}} \beta(t)>$ $\gamma+\mu$ holds; Bai and Zhou [18], Bai et al. [19], and Liu [20] studied the existence of periodic solutions for a periodically forced SIR model with saturated incidence rates. By persistence theory, Zhang and Zhao [21] studied a periodic epidemic model in a patchy environment; Sun et al. [22] studied the SEI model with seasonality comprehensively; Rebelo et al. [23] extended these results to some delay differential equations and partial differential equations.

When $f(I)$ in (1.1) is a non-smooth function, to the best of our knowledge, there are no results on the existence of periodic solutions. The methods we mentioned above cannot deal with the non-smooth righthand sides directly.

In this paper, we use an integral version of Leray-Schauder degree theory under Katriel's frame to prove the existence of periodic solutions for our SIR model. Some numerical simulations are presented to illustrate the effectiveness of such media coverage. Our main results are as follows.

Theorem 1.1 Whenever $\mathcal{R}_{0}>e^{\alpha}$, there exists at least one T-periodic solution $(S(t), I(t)$, $R(t))$ of (1.1)-(1.2), all of whose components are positive.

In [24], Liu and Xiao consider the non-periodic coefficient SIR model with

$$
f(I)= \begin{cases}e^{-m I}, & I<I_{c}, \\ e^{-m I_{c}}, & I>I_{c},\end{cases}
$$

where $m>0$ is a factor of influence and $I_{c}$ is a critical level. Using the same method as in Theorem 1.1, we can present the following theorem without a proof.

Theorem 1.2 Whenever $\mathcal{R}_{0}>e^{m I_{c}}$, there exists at least one T-periodic solution $(S(t), I(t)$, $R(t))$ of (1.1) and (1.3), all of whose components are positive. 
This paper is organized as follows. In Section 2, we study the properties of the homotopy equation from the classical autonomous SIR model to our SIR model. In Section 3, we construct an equivalent integral equation and define a completely continuous operator. In Section 4, we prove the main theorem by Leray-Schauder degree theory. In Section 5, some numerical simulations are presented to illustrate the effectiveness of such media coverage.

\section{Homotopy equation and suitable domain}

In the rest of this paper, we assume that $f(I)$ has the expression (1.2). Observing system (1.1), we have $\frac{d S}{d t}+\frac{d I}{d t}+\frac{d R}{d t} \equiv 0$. Since $S(t), I(t), R(t)$ are fractions of the population, we have $S(t)+I(t)+R(t)=1$ for all $t$. Because $R$ does not appear in the first two equations in (1.1), it is sufficient to consider the existence of periodic solutions of following systems:

$$
\left\{\begin{array}{l}
\frac{d S(t)}{d t}=\mu-\beta(t) f(I) S I-\mu S, \\
\frac{d I(t)}{d t}=\beta(t) f(I) S I-(\mu+\gamma) I,
\end{array}\right.
$$

with

$$
S(t)>0, \quad I(t)>0, \quad S(t)+I(t)<1 .
$$

In order to prove the existence of periodic solutions of (2.1), we consider the following homotopy system:

$$
\left\{\begin{array}{l}
\frac{d S(t)}{d t}=\mu-\bar{\beta} S I-\mu S-\lambda(\beta(t) f(I) S I-\bar{\beta} S I), \\
\frac{d I(t)}{d t}=\bar{\beta} S I-(\mu+\gamma) I+\lambda(\beta(t) f(I) S I-\bar{\beta} S I),
\end{array}\right.
$$

where $\lambda \in[0,1]$. Let

$$
D:=\{(S, I) \in C[0, T] \times C[0, T] \mid S(t)>0, I(t)>0, S(t)+I(t)<1\} .
$$

Lemma 2.1 $\bar{D}$ is an invariant region with respect to (2.2). The disease-free equilibrium $\left(S_{0}, I_{0}\right)=(1,0)$ is the unique periodic solution of $(2.2)$ satisfying $(S, I) \in \partial D$ for any $\lambda \in[0,1]$.

Proof First, we will prove that $\bar{D}$ is an invariant region. In fact, it follows from model (2.2) that

$$
\left.\frac{d S}{d t}\right|_{S=0}=\mu>0,\left.\quad \frac{d I}{d t}\right|_{I=0}=0,\left.\quad \frac{d(S+I)}{d t}\right|_{S+I=1}=-\gamma I \leq 0 .
$$

Second, we will prove that the disease-free equilibrium $\left(S_{0}, I_{0}\right)=(1,0)$ is the unique periodic solution of (2.2) satisfying $(S, I) \in \partial D$. We assume that $(S, I) \in \partial D$ is a solution of (2.2), which means that at least one of the following conditions holds:

(i) There exists $t_{0} \in[0, T]$ such that $I\left(t_{0}\right)=0$.

(ii) There exists $t_{0} \in[0, T]$ such that $S\left(t_{0}\right)=0$.

(iii) There exists $t_{0} \in[0, T]$ such that $S\left(t_{0}\right)+I\left(t_{0}\right)=1$.

We now consider each of these three cases:

In the case of (i), we have $I\left(t_{0}\right)=0$ and $I^{\prime}\left(t_{0}\right)=0$, which implies $I \equiv 0$. Thus, the only possible periodic solution of $S^{\prime}=\mu(1-S)$ is $S \equiv 1$. 
In the case of (ii), we have $S\left(t_{0}\right)=0$ and $S^{\prime}\left(t_{0}\right)=\mu>0$. Thus, it is easy to obtain that $S(t)<0$ for $t<t_{0}$ sufficiently close to $t_{0}$, which contradicts the fact that $\bar{D}$ is an invariant region.

In the case of (iii), we get

$$
(S+I)^{\prime}\left(t_{0}\right)=\mu\left(1-S\left(t_{0}\right)-I\left(t_{0}\right)\right)-\gamma I\left(t_{0}\right)=-\gamma I\left(t_{0}\right) \leq 0 .
$$

Because the case of $I\left(t_{0}\right)=0$ has been discussed, we only discuss the case $S\left(t_{0}\right)+I\left(t_{0}\right)=1$, $(S+I)^{\prime}\left(t_{0}\right)<0$, which contradicts the fact that $\bar{D}$ is an invariant region.

To use the continuity method, we need to choose a bounded open set $U \subseteq D$ such that there is no solution $(S, I)$ of $(2.2)$ satisfying $(S, I) \in \partial U$ for any $\lambda \in[0,1]$. Since $\left(S_{0}, I_{0}\right) \in$ $\partial D$ is a solution of $(2.2)$, we need to exclude $\left(S_{0}, I_{0}\right)$ from the boundary of $U$ we chose. Following the idea of Katriel [16], we take $U$ to be the open subset of $D$ given by

$$
U=\left\{(S, I) \in D \mid \min _{t \in[0, T]} S(t)<\delta\right\}
$$

where $\delta \in(0,1)$ is to be fixed.

Remark 2.2 For fixed $t_{0}, U$ is an open set in $\mathbb{R}^{2}$. Furthermore, for any $t \in[0, T], U$ with norm $\|(S, I)\|=\max _{t \in[0, T]}(|S(t)|+|I(t)|)$ is an open set in $C[0, T] \times C[0, T]$.

Lemma 2.3 Let $\mathcal{R}_{0}>e^{\alpha}$. If we choose $\delta \in\left(\frac{e^{\alpha}}{\mathcal{R}_{0}}, 1\right)$, then there is no solution $(S, I)$ of $(2.2)$ with $(S, I) \in \partial U$ for any $\lambda \in[0,1]$.

Proof Suppose $(S, I) \in \partial U$. Then either $(S, I) \in \partial D$ or $(S, I) \in D$ and

$$
\min _{t \in[0, T]} S(t)=\delta
$$

In the first case, Lemma 2.1 and the fact that $\left(S_{0}, I_{0}\right) \notin \partial U$ imply that $(S, I)$ is not a solution of (2.2).

In the second case, we can infer that $I(t)>0$ and $S(t) \geq \delta, \forall t \in[0, T]$. If $(S, I)$ is a solution of (2.2), we can divide the second equation of (2.2) by $I$. Integrating over $[0, T]$, we obtain that

$$
\frac{1}{T} \int_{0}^{T} \bar{\beta} S d t-\mu-\gamma+\frac{1}{T} \int_{0}^{T} \lambda(\beta(t) S f(I)-\bar{\beta} S) d t=0 .
$$

Thus

$$
\begin{aligned}
\mu+\gamma & =\frac{1}{T} \int_{0}^{T}(1-\lambda) \bar{\beta} S+\lambda \beta(t) S f(I) d t \\
& \geq \frac{\delta}{T} \int_{0}^{T}(1-\lambda) \bar{\beta}+\lambda \beta(t) f(I) d t \\
& \geq \delta e^{-\alpha} \bar{\beta} .
\end{aligned}
$$


By the assumption $\delta>\frac{e^{\alpha}}{\mathcal{R}_{0}}$, we have

$$
\mu+\gamma<\delta e^{-\alpha} \bar{\beta}
$$

which is a contradiction.

\section{Existence of periodic solutions}

3.1 Equivalent integral equation and completely continuous operator

We rewrite (2.2) as

$$
\frac{d}{d t}\left(\begin{array}{l}
S \\
I
\end{array}\right)+\left(\begin{array}{cc}
\mu & 0 \\
0 & \mu+\gamma
\end{array}\right)\left(\begin{array}{l}
S \\
I
\end{array}\right)=\left(\begin{array}{l}
F_{1}(S, I, \lambda) \\
F_{2}(S, I, \lambda)
\end{array}\right)
$$

where $F_{1}(S, I, \lambda)=\mu-\bar{\beta} S I-\lambda(\beta(t) f(I) S I-\bar{\beta} S I)$, and $F_{2}(S, I, \lambda)=\bar{\beta} S I+\lambda(\beta(t) f(I) S I-\bar{\beta} S I)$. If $\Phi(t)$ is the fundamental solution matrix of

$$
\frac{d}{d t}\left(\begin{array}{l}
S \\
I
\end{array}\right)+\left(\begin{array}{cc}
\mu & 0 \\
0 & \mu+\gamma
\end{array}\right)\left(\begin{array}{l}
S \\
I
\end{array}\right)=0
$$

satisfying $\Phi(0)$ = Id, we have

$$
\Phi(t)=\left(\begin{array}{cc}
e^{-\mu t} & 0 \\
0 & e^{-(\mu+\gamma) t}
\end{array}\right)
$$

Then (3.1) can be transformed into the equivalent integral equation

$$
\left(\begin{array}{l}
S \\
I
\end{array}\right)(t)=\Phi(t)\left(\left(\begin{array}{c}
S(0) \\
I(0)
\end{array}\right)+\int_{0}^{t} \Phi^{-1}(\tau)\left(\begin{array}{l}
F_{1}(S, I, \lambda) \\
F_{2}(S, I, \lambda)
\end{array}\right) d \tau\right) .
$$

If $S, I$ is a $T$-periodic solution of (3.1), then

$$
(I-\Phi(T))\left(\begin{array}{c}
S(0) \\
I(0)
\end{array}\right)=\Phi(T) \int_{0}^{T} \Phi^{-1}(\tau)\left(\begin{array}{l}
F_{1}(S, I, \lambda) \\
F_{2}(S, I, \lambda)
\end{array}\right) d \tau
$$

Since $(I-\Phi(T))$ is invertible, we have

$$
\left(\begin{array}{c}
S(0) \\
I(0)
\end{array}\right)=(I-\Phi(T))^{-1} \Phi(T) \int_{0}^{T} \Phi^{-1}(\tau)\left(\begin{array}{c}
F_{1}(S, I, \lambda) \\
F_{2}(S, I, \lambda)
\end{array}\right) d \tau
$$

Substituting (3.4) into (3.2), we have

$$
\begin{aligned}
\left(\begin{array}{l}
S \\
I
\end{array}\right)(t)= & \Phi(t)(I-\Phi(T))^{-1} \Phi(T) \int_{0}^{T} \Phi^{-1}(\tau)\left(\begin{array}{l}
F_{1}(S, I, \lambda) \\
F_{2}(S, I, \lambda)
\end{array}\right) d \tau \\
& +\Phi(t) \int_{0}^{t} \Phi^{-1}(\tau)\left(\begin{array}{l}
F_{1}(S, I, \lambda) \\
F_{2}(S, I, \lambda)
\end{array}\right) d \tau .
\end{aligned}
$$


Define an operator $P_{\lambda}: C[0, T] \times C[0, T] \rightarrow C[0, T] \times C[0, T]$ such that

$$
\begin{aligned}
P_{\lambda}[(S, I)](t)= & \Phi(t)(\mathrm{Id}-\Phi(T))^{-1} \Phi(T) \int_{0}^{T} \Phi^{-1}(\tau)\left(\begin{array}{l}
F_{1}(S, I, \lambda) \\
F_{2}(S, I, \lambda)
\end{array}\right) d \tau \\
& +\Phi(t) \int_{0}^{t} \Phi^{-1}(\tau)\left(\begin{array}{c}
F_{1}(S, I, \lambda) \\
F_{2}(S, I, \lambda)
\end{array}\right) d \tau .
\end{aligned}
$$

Lemma 3.1 $P_{\lambda}$ in (3.6) is a completely continuous operator.

Proof Since $f(I)$ is a non-smooth but continuous function, the operator $P_{\lambda}$ is continuous with respect to $S$ and $I$. Since $\beta(t), \Phi(t)$ and $\Phi^{-1}(t)$ are all bounded in $[0, T], S$ and $I$ are bounded on $U, e^{-\alpha} \leq f(I) \leq 1$ on $U$, it is easy to see that the operator $P_{\lambda}$ in (3.6) is uniformly bounded and equicontinuous, which implies $P_{\lambda}$ in (3.6) is a completely continuous operator.

\subsection{Main results}

We recall that the existence of a periodic solution $(S, I)$ of (3.5) can be assured by LeraySchauder degree theory $[25]$ if the following conditions hold:

(1) $\left(\operatorname{Id}-P_{\lambda}\right)(S, I) \neq 0$ for all $(S, I) \in \partial U, \lambda \in[0,1]$,

(2) $\operatorname{deg}\left(\operatorname{Id}-P_{0}, U, 0\right) \neq 0$.

By Lemma 2.3, there are no solutions $(S, I)$ of $(2.2)$ with $(S, I) \in \partial U, \lambda \in[0,1]$. Now we prove that $\operatorname{deg}\left(\mathrm{Id}-P_{0}, U, 0\right) \neq 0$.

Lemma 3.2 For $\lambda=0,(2.2)$ has only one periodic solution in $U$, which is endemic equilibrium: $\left(S^{*}, I^{*}\right)=\left(\frac{\gamma+\mu}{\bar{\beta}}, \mu\left(\frac{1}{\gamma+\mu}-\frac{1}{\bar{\beta}}\right)\right)$.

Proof When $\lambda=0,(2.2)$ is an autonomous system. $\mu(1-S)-\bar{\beta} S I$ and $\bar{\beta} S I-(\mu+\gamma) I$ are both $C^{1}$ in $D$. If we set the Dulac function to be $h=\frac{1}{S I}$ in $D$, we have

$$
\frac{\partial(h(\mu(1-S)-\bar{\beta} S I))}{\partial S}+\frac{\partial(h(\bar{\beta} S I-(\mu+\gamma) I))}{\partial I}=-\frac{\mu}{S^{2} I}<0,
$$

so there is no closed orbit in $D$.

Since $\frac{e^{\alpha}}{\mathcal{R}_{0}}<\delta<1, \gamma, \mu$ and $\bar{\beta}$ are positive constant, we have $0<S^{*}<\frac{\delta}{e^{\alpha}}<\delta$ and $0<I^{*}<$ $\frac{\mu}{\gamma+\mu}<1$. For $\lambda=0$, it is easy to calculate that $\left(S^{*}, I^{*}\right)$ is the unique constant periodic solution in $U$, which implies that (2.2) has only one periodic solution in $U$.

Lemma 3.3 [26] Let $\Omega$ be a bounded open set in the Banach space X. Assume that a completely continuous field $f=\operatorname{Id}-F: \bar{\Omega} \rightarrow X$ has no zero points on $\partial \Omega$, and there are only finite zero points $x_{1}, x_{2}, \ldots, x_{n}$ in $\Omega$. Then we have the index formula

$$
\operatorname{deg}(f, \Omega, 0)=\sum_{i=1}^{n} \operatorname{index}\left(f, x_{i}\right)
$$

Lemma 3.4 [26] Let $\Omega$ be a bounded open set in the Banach space X. $x_{0}$ is the zero point of a completely continuous field $f=\operatorname{Id}-F: \bar{\Omega} \rightarrow X$ in $\Omega$. We assume that $f$ is Frechet differentiable at $x_{0}$ and 1 is not the eigenvalue of $A=F^{\prime}\left(x_{0}\right)$. Then $x_{0}$ is an isolated zero point 
off, and

$$
\operatorname{index}\left(f, x_{0}\right)=\operatorname{index}(\operatorname{Id}-A, 0)=(-1)^{\beta},
$$

where $\beta=\sum_{\lambda_{j}>1 \mid \lambda_{j} \in \sigma(A)} \beta_{j}$ and $\beta_{j}=\operatorname{dim} \bigcup_{k=1}^{\infty} \operatorname{Ker}\left(\lambda_{j} I-A\right)^{k}$.

Define the operator

$$
\begin{aligned}
& D P_{0}\left[\left(S^{*}, I^{*}\right)\right] \circ(V, W)(t) \\
& =\Phi(t)(\operatorname{Id}-\Phi(T))^{-1} \Phi(T) \int_{0}^{T} \Phi^{-1}(\tau)\left(\begin{array}{c}
-\bar{\beta}\left(I^{*} V+S^{*} W\right) \\
\bar{\beta}\left(I^{*} V+S^{*} W\right)
\end{array}\right) d \tau \\
& \quad+\Phi(t) \int_{0}^{t} \Phi^{-1}(\tau)\left(\begin{array}{c}
-\bar{\beta}\left(I^{*} V+S^{*} W\right) \\
\bar{\beta}\left(I^{*} V+S^{*} W\right)
\end{array}\right) d \tau .
\end{aligned}
$$

Obviously,

$$
\left\|P_{0}\left[\left(S^{*}+V, I^{*}+W\right)\right]-P_{0}\left[\left(S^{*}, I^{*}\right)\right]-D P_{0}\left[\left(S^{*}, I^{*}\right)\right] \circ(V, W)\right\|=o[(V, W)] .
$$

Thus, $D P_{0}\left[\left(S^{*}, I^{*}\right)\right]$ is the Frechet derivative of $P_{0}[(S, I)]$.

Lemma 3.5 1 is not the eigenvalue of $D P_{0}\left(S^{*}, I^{*}\right)$.

Proof Let $\lambda$ be the eigenvalue of operator $D P_{0}\left(S^{*}, I^{*}\right)$ :

$$
D P_{0}\left[\left(S^{*}, I^{*}\right)\right] \circ(V, W)(t)=\lambda(V, W)
$$

Multiplying $\Phi^{-1}(t)$ by both sides of (3.11) and taking the derivative with respect to $t$, we have

$$
\Phi^{-1}(t)\left(\begin{array}{c}
-\bar{\beta}\left(I^{*} V+S^{*} W\right) \\
\bar{\beta}\left(I^{*} V+S^{*} W\right)
\end{array}\right)=\frac{d}{d t}\left(\lambda \Phi^{-1}(t)\left(\begin{array}{c}
V \\
W
\end{array}\right)\right)
$$

which is equal to

$$
\lambda\left(\begin{array}{c}
\dot{V} \\
\dot{W}
\end{array}\right)=\left(\begin{array}{cc}
-\bar{\beta} I^{*}-\lambda \mu & -\bar{\beta} S^{*} \\
\bar{\beta} I^{*} & \bar{\beta} S^{*}-\lambda \mu-\lambda \gamma
\end{array}\right)\left(\begin{array}{c}
V \\
W
\end{array}\right) .
$$

If 1 is an eigenvalue of $D P_{0}\left(S^{*}, I^{*}\right)$, we have

$$
\frac{d}{d t}\left(\begin{array}{c}
V \\
W
\end{array}\right)=\left(\begin{array}{cc}
-\mu \mathcal{R}_{0} & -(\gamma+\mu) \\
\mu\left(\mathcal{R}_{0}-1\right) & 0
\end{array}\right)\left(\begin{array}{c}
V \\
W
\end{array}\right) .
$$

The characteristic polynomial of the above matrix is

$$
p(x)=x^{2}+\mu \mathcal{R}_{0} x+(\gamma+\mu) \mu\left(\mathcal{R}_{0}-1\right) .
$$


It is easy to calculate that $p(0)>0$, and $p(\omega i) \neq 0$ for $\omega \in \mathbb{R}$, which means that the matrix has no imaginary or 0 eigenvalues, so that (3.14) has no periodic solutions except $(V, W)=$ $(0,0)$, which is a contradiction to (3.11).

Now we will finish to prove that $\operatorname{deg}\left(\mathrm{Id}-P_{0}, U, 0\right) \neq 0$. Id $-P_{0}$ is a completely continuous field, 1 is not the eigenvalue of $D P_{0}\left(S^{*}, I^{*}\right)$. By Lemma 3.4, we have index $\left(\operatorname{Id}-P_{0},\left(S^{*}, I^{*}\right)\right)=$ $(-1)^{\beta} \neq 0$. Since we have proved that $\left(\operatorname{Id}-P_{0}\right)(S, I) \neq 0$ for all $(S, I) \in \partial U$, and $\left(S^{*}, I^{*}\right)$ is the unique zero point in $U$, by Lemma 3.3 we have

$$
\operatorname{deg}\left(\operatorname{Id}-P_{0}, U, 0\right)=\operatorname{index}\left(\operatorname{Id}-P_{0},\left(S^{*}, I^{*}\right)\right) \neq 0 .
$$

Finally, by Leray-Schauder degree theory, we obtain

$$
\operatorname{deg}\left(\operatorname{Id}-P_{1}, U, 0\right)=\operatorname{deg}\left(\operatorname{Id}-P_{0}, U, 0\right) \neq 0 .
$$

Thus, there exists at least one positive periodic solutions for a seasonally forced SIR model with impact of media coverage.

\section{Simulation}

In this section, we present some numerical examples to illustrate the effectiveness of such media coverage. Furthermore, we show how various parameters influence the solutions of our SIR model.

With the period $T=2 \pi$ of the forcing representing one year, we take $\gamma=14 \frac{2 \pi}{365}$ corresponding to a two-week infectious period. We set $\bar{\beta}=4 \gamma, \mu=\frac{0.5}{2 \pi}, \beta(t)=\bar{\beta}(1+0.8 \cos (t))$, $I_{c}=0.15$ and $\delta=0.05$. Let $[0,2 \pi]$ be divided into $k=200$ intervals equally. Given the initial point $\left(S^{* *}, I^{* *}\right)=\left(\frac{\mu+\gamma}{\bar{\beta}}, \frac{\mu}{\mu+\gamma}-\frac{\mu}{\bar{\beta}}\right)$, which is the endemic equilibrium of SIR model without periodic transmission rate and impact of media coverage. The periodic solutions of system (1.1) can be solved by the Newton iteration method.

In Figure 1, we make ten steps of Newton iteration to get the approximate infective population and susceptible population of system (1.1) with different $\alpha$. Obviously, the infective

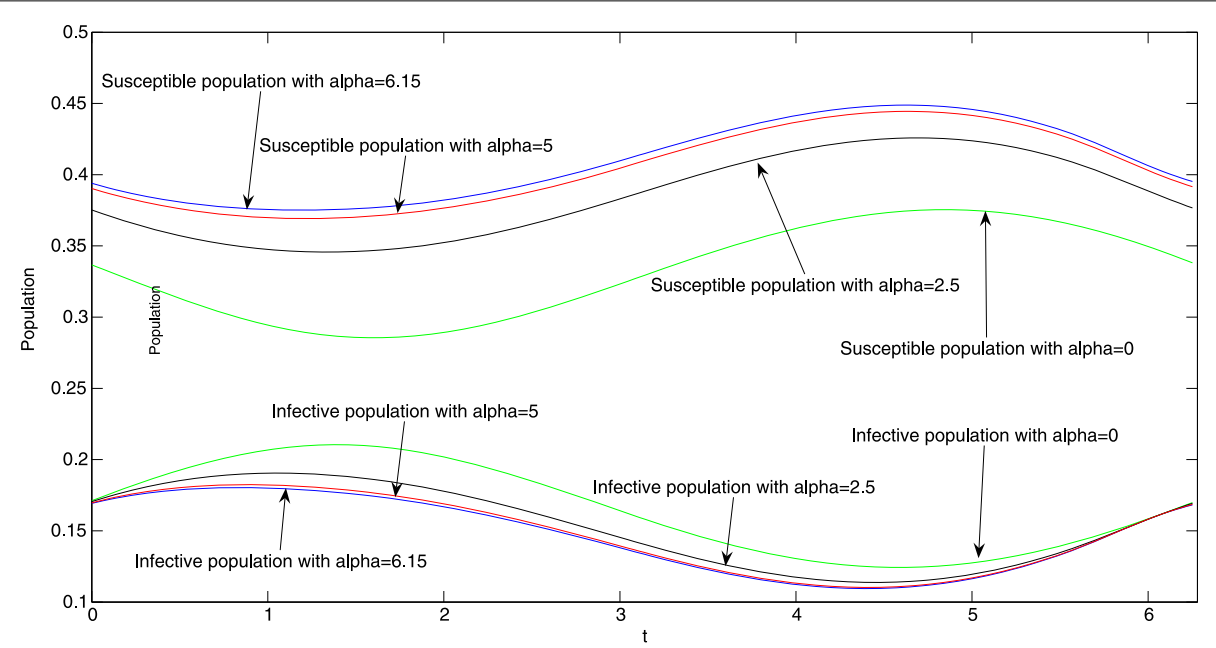

Figure 1 Infective population and susceptible population with $\alpha=0, \alpha=2.5, \alpha=5$ and $\alpha=6.15$. 


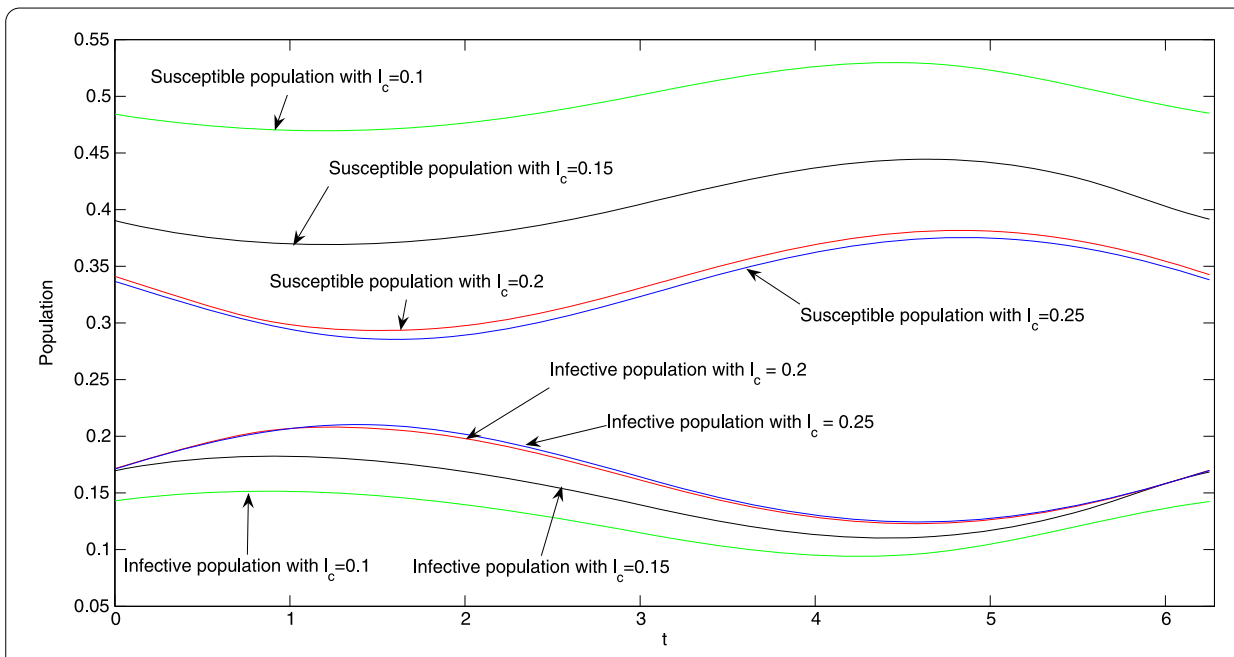

Figure 2 Infective population and susceptible population with $I_{c}=0.1, I_{c}=0.15, I_{c}=0.2$ and $I_{c}=0.25$.

population of system (1.1) with impact of media coverage $(\alpha>0)$ is lower than the infective population of system (1.1) without impact of media coverage $(\alpha=0)$. The effectiveness of impact of media coverage grows as $\alpha$ grows. The solutions in both cases are locally stable and the error is about $10^{-6}$.

In Figure 2, we fix $\alpha=5$ and make ten steps of Newton iteration to get the approximate infective population and susceptible population of system (1.1) with different $I_{c}$. Obviously, the infective population of system (1.1) becomes smaller as $I_{c}$ decreases. The solutions in both cases are locally stable and the error is about $10^{-6}$.

In a word, it is effective to reduce the infective population by media coverage.

\section{Conclusion}

In this paper, we study the existence of positive periodic solutions for a seasonally forced SIR model with impact of media coverage. This paper can be divided into two parts. In the first part, we construct a homotopy equation from an autonomous system to our SIR model. Using Leray-Schauder degree theory, we establish a new result on the existence of at least one positive periodic solution for our SIR model. In the final part, some numerical simulations are presented to illustrate the effect of media coverage.

Competing interests

The authors declare that they have no competing interests.

Authors' contributions

All authors contributed equally to the writing of this paper. All authors read and approved the final manuscript.

\section{Author details}

${ }^{1}$ Center for Mathematics and Interdisciplinary Sciences, and School of Mathematics and Statistics, Northeast Normal University, Changchun, 130024, P.R. China. ${ }^{2}$ School of Basic Science, Changchun University of Technology, Changchun, 130012, P.R. China. ${ }^{3}$ College of Mathematics, Jilin University, Changchun, 130012, P.R. China.

\section{Acknowledgements}

The authors would like to express their sincere gratitude to Professor Yong Li for his enthusiastic guidance and constant encouragement. Jian Zu was supported by NSFC (Grant No. 11401089). 
References

1. Altizer, S, et al.: Seasonality and the dynamics of infectious diseases. Ecol. Lett. 9, 467-484 (2006)

2. Grassly, NC, Fraser, C: Seasonal infectious disease epidemiology. Proc. - Royal Soc., Biol. Sci. 273, 2541-2550 (2006)

3. Hethcote, HW, Levin, SA: Periodicity in epidemiological models. In: Applied Mathematical Ecology (Trieste, 1986). Biomathematics, vol. 18, pp. 193-211. Springer, Berlin (1989)

4. Grossman, Z, Gumowski, I, Dietz, K: The incidence of infectious diseases under the influence of seasonal fluctuations analytical approach. In: Nonlinear Systems and Applications (Proc. Internat. Conf., Univ. Texas, Arlington, Tex., 1976), pp. 525-546. Academic Press, New York (1977)

5. Lou, YJ, Zhao, XQ: Threshold dynamics in a time-delayed periodic SIS epidemic model. Discrete Contin. Dyn. Syst., Ser. B 12(1), 169-186 (2009)

6. Lou, YJ, Zhao, XQ: A climate-based malaria transmission model with structured vector population. SIAM J. Appl. Math. 70(6), 2023-2044 (2010)

7. Ma, JL, Ma, ZE: Epidemic threshold conditions for seasonally forced SEIR models. Math. Biosci. Eng. 3(1), 161-172 (2006)

8. Earn, DJ, Rohani, P, Bolker, BM, Grenfell, BT: A simple model for complex dynamical transitions in epidemics. Science 287(5453), 667-670 (2000)

9. Liu, RS, Wu, JH, Zhu, HP: Media/psychological impact on multiple outbreaks of emerging infectious diseases. Comput. Math. Methods Med. 8(3), 153-164 (2007)

10. Cui, JA, Sun, YH, Zhu, HP: The impact of media on the control of infectious diseases. J. Dyn. Differ. Equ. 20(1), 31-53 (2008)

11. Cui, JA, Tao, X, Zhu, HP: An SIS infection model incorporating media coverage. Rocky Mt. J. Math. 38(5), 1323-1334 (2008)

12. Sun, CJ, Yang, W, Arino, J, Khan, K: Effect of media-induced social distancing on disease transmission in a two patch setting. Math. Biosci. 230(2), 87-95 (2011)

13. Li, YF, Cui, JA: The effect of constant and pulse vaccination on SIS epidemic models incorporating media coverage. Commun. Nonlinear Sci. Numer. Simul. 14(5), 2353-2365 (2009)

14. Cai, LM, Li, XZ: Analysis of a SEIV epidemic model with a nonlinear incidence rate. Appl. Math. Model. 33(7), 2919-2926 (2009)

15. Wang, AL, Xiao, YN: A Filippov system describing media effects on the spread of infectious diseases. Nonlinear Anal. Hybrid Syst. 11, 84-97 (2014)

16. Katriel, G: Existence of periodic solutions for the periodically forced SIR model. Nelīnïnī Koliv. 16(3), 359-366 (2013)

17. Jódar, L, Villanueva, RJ, Arenas, A: Modeling the spread of seasonal epidemiological diseases: theory and applications. Math. Comput. Model. 48(3-4), 548-557 (2008)

18. Bai, ZG, Zhou, YC: Existence of two periodic solutions for a non-autonomous SIR epidemic model. Appl. Math. Model. 35(1), 382-391 (2011)

19. Bai, ZG, Zhou, YC, Zhang, TL: Existence of multiple periodic solutions for an SIR model with seasonality. Nonlinear Anal. 74(11), 3548-3555 (2011)

20. Liu, ZJ: Dynamics of positive solutions to SIR and SEIR epidemic models with saturated incidence rates. Nonlinear Anal., Real World Appl. 14(3), 1286-1299 (2013)

21. Zhang, F, Zhao, XQ: A periodic epidemic model in a patchy environment. J. Math. Anal. Appl. 325(1), 496-516 (2007)

22. Sun, GQ, Bai, ZG, Zhang, ZK, Zhou, T, Jin, Z: Positive periodic solutions of an epidemic model with seasonality. Sci. World J. 2013, Article ID 470646 (2013)

23. Rebelo, C, Margheri, A, Bacaër, N: Persistence in some periodic epidemic models with infection age or constant periods of infection. Discrete Contin. Dyn. Syst., Ser. B 19(4), 1155-1170 (2014)

24. Liu, YY, Xiao, YN: An epidemic model with saturated media/psychological impact. Appl. Math. Mech. 34(4), 399-407 (2013)

25. Zeidler, E: Nonlinear Functional Analysis and Its Applications. I. Fixed-Point Theorems. Springer, New York (1986); Translated from the German by Peter R. Wadsack

26. Chang, KC: Methods in Nonlinear Analysis. Springer Monographs in Mathematics. Springer, Berlin (2005)

\section{Submit your manuscript to a SpringerOpen ${ }^{\circ}$ journal and benefit from:}

- Convenient online submission

Rigorous peer review

- Immediate publication on acceptance

- Open access: articles freely available online

- High visibility within the field

- Retaining the copyright to your article 